\title{
Conventional and rapid methods for identification of Staphylococcus aureus from clinical specimens
}

\author{
Nneoma Confidence JeanStephanie Anyanwu ${ }^{1, ~ *}$, Walter Chinaka John ${ }^{2}$ \\ ${ }^{1}$ Department of Microbiology, Faculty of Sciences, Ahmadu Bello University, Zaria, Nigeria \\ ${ }^{2}$ Federal College of Forestry, Jos, Plateau State, Nigeria
}

Email address:

anyanwunneomaconfidence@gmail.com (N. C. J. Anyanwu)

\section{To cite this article:}

Nneoma Confidence JeanStephanie Anyanwu, Walter Chinaka John. Conventional and Rapid Methods for Identification of Staphylococcus aureus from Clinical Specimens. American Journal of Biomedical and Life Sciences. Vol. 1, No. 3, 2013 , pp. 41-43. doi: 10.11648/j.ajbls.20130103.11

\begin{abstract}
Staphylococcus aureus is a facultative anaerobic Gram-positive coccal bacterium whose incidence ranges from skin, soft tissue, respiratory, bone, joint, endovascular to wound infections. The purpose of this study was to identify Staphylococcus aureus from clinical specimens using routine conventional and rapid tests. Gram staining, catalase test, coagulase test, DNase test, haemolysis on blood agar and Microgen ${ }^{\text {TM }}$ STAPH-ID kit tests were carried out. A total of 125 Gram positive cocci were tested. The Gram staining technique yielded 100 (80.00\%) Staphylococcus spp (Gram positive cocci in clusters). $89(71.20 \%$ ) isolates were positive to haemolysis on blood agar. Mannitol Salt Agar, DNase agar and Catalase test correctly identified $69(55.2 \%)$ of the Gram positive cocci to be $S$. aureus as was confirmed by the Microgen ${ }^{\mathrm{TM}}$ STAPH-ID kit test. Coagulase test yielded 66 (52.8\%) positive results. The Microgen ${ }^{\mathrm{TM}}$ STAPH-ID kit test identified three non-coagulase Staphylococcus aureus isolates. The Microgen ${ }^{\text {TM }}$ STAPH-ID kit test was the most reliable of the tests, with accuracy comparable to any other rapid test. However, it is the most expensive of the tests. This study established that conventional tests can be used for direct identification of $S$. aureus to species level if the battery of tests is increased.
\end{abstract}

Keywords: Staphylococcus aureus, Conventional Tests, Rapid Tests, Coagulase Test, DNase Test, Microgen $^{\text {TM }}$ STAPH-ID

\section{Introduction}

Staphylococcus aureus is a facultative anaerobic Grampositive coccal bacterium whose incidence ranges from skin, soft tissue, respiratory, bone, joint, endovascular to wound infections (1). Rapid and direct identification of $S$. aureus is crucial for proper management of patients with skin infections, abscesses, septicemia/bacteremia, gastroenteritis, endocarditis, toxic shock syndrome and certain food intoxications $(2,3)$.

In developing countries, phenotypic tests are routinely used in the diagnosis of staphylococcal infections, in which coagulase tests are usually confirmatory for $S$. aureus $(4,5)$. Staphylococcus aureus is usually isolated on non-specific media (e.g. blood agar) and then presumptively identified before definitive overnight characterization (6). In an attempt to achieve presumptive isolation in a single step, mannitol salt agar (MSA) was developed in 1945 for the selective isolation of pathogenic staphylococci in the clinical microbiology laboratory (7). The growth and production of yellow colonies, due to the high salt content of the medium and fermentation of mannitol, is regarded as a presumptive tool in the identification of $S$. aureus. It is also described as a characteristic for the differentiation of coagulase-positive staphylococci from coagulase-negative staphylococci (CoNS) (8). However, there are reports that some CoNS can also produce yellow colonies on MSA (9, 10,11). Single phenotypic tests are inefficient for the identification of $S$. aureus. However, a combination of MSA and DNase improves the tube coagulase test (12).

The purpose of this study was to identify Staphylococcus aureus from clinical specimens using routine conventional and rapid tests.

\section{Materials and Methods}

\subsection{Specimens}

A total of 100 clinical specimens (ranging from swabs to 
internal fluids except blood and cerebrospinal fluid) positive for Gram positive cocci (GPC) were included (Table1). Specimens were collected from patients in hospitals in Zaria, Nigeria, between April and October, 2012. Specimens collected were transported immediately to the postgraduate Clinical Microbiology laboratory of the Department of Microbiology, Ahmadu Bello University, Zaria, were the tests were performed.

\subsection{Identification of Isolates using Conventional Tests}

Isolates were incubated at $37^{\circ} \mathrm{C}$ for $18-24$ hours on blood agar (Oxoid, Cambridge, UK) and observed for haemolysis. Gram staining was carried out on presumptive isolates. Single colonies of Gram positive cocci were then tested with catalase test, coagulase test, DNase test and growth on MSA. Sequel testing of the isolates was further performed beginning with MSA, followed by DNase and finally Tube CoagulaseTest, to evaluate the performance of individual tests. S. aureus ATCC 25923 and Staphylococcus epidermidis ATCC 12228 were used as positive control.

\subsection{Characterisation of Isolates using Microgen ${ }^{\mathrm{TM}}$ STAPH-ID System}

The isolates suspected to be Staphylococcus spp were further identified with Microgen ${ }^{\text {TM }}$ MID-69 microwell test strips. A single colony each, from 18-24 hours culture was emulsified in suspending medium supplied in the kit. The adhesive tapes sealing the microwell test strips were carefully peeled off and kept safe. Sterile Pasteur pipette was used to add 3-4 drops (approximately 100 $\mu \mathrm{L}$ ) of bacterial suspension to each well of the strips, after which wells 10 and 11 were overlaid with 3-4 drops of mineral oil.
The microwell test strips were then sealed with the adhesive tapes removed earlier and incubated at $35-37{ }^{\circ} \mathrm{C}$ for 18-24 hours. The results were read with the aid of the Microgen ${ }^{\mathrm{TM}}$ software database (containing the colour chart and substrate reference table) after adding the necessary reagents (PYR and Nitrate $A$ and $B$ ).

\section{Results}

Table 1 shows the isolates screened in this study, Table 2 shows $S$. aureus isolated using conventional tests, Table 3 shows Microgen STAPHYLOCOCCUS-ID 12 test result for some $S$. aureus isolates

Table 1: Isolates Screened in this Study

\begin{tabular}{ll}
\hline Organism & Number of isolates obtained \\
\hline Staphylococcus aureus & 69 \\
Staphylococcus epidermidis & 31 \\
Other Gram positive cocci & 25 \\
Total & 125 \\
\hline
\end{tabular}

Table 2: Isolation of S. aureus Using Conventional Tests (including Sequel Testing).

\begin{tabular}{llll}
\hline Tests conducted & $\begin{array}{l}\text { Number of } \\
\text { isolates } \\
\text { screened }\end{array}$ & $\begin{array}{l}\text { Number of } \\
\text { positive } \\
\text { isolates }\end{array}$ & $\begin{array}{l}\text { Percentage } \\
\text { positive (\%) }\end{array}$ \\
\hline $\begin{array}{l}\text { Gram Staining } \\
\text { Haemolysis on }\end{array}$ & 125 & 100 & 80.00 \\
$\begin{array}{l}\text { Blood Agar } \\
\text { Yellow colonies }\end{array}$ & 125 & 89 & 71.20 \\
$\begin{array}{l}\text { on MSA } \\
\text { Clear zone on }\end{array}$ & 125 & 69 & 55.20 \\
$\begin{array}{l}\text { DNase Agar } \\
\text { Catalase Test }\end{array}$ & 125 & 69 & 55.20 \\
Coagulase Test & 125 & 69 & 55.20 \\
\hline
\end{tabular}

Table 3: Microgen STAPHYLOCOCCUS-ID 12 Test Result for Some Isolates

\begin{tabular}{|c|c|c|c|c|c|c|c|c|c|c|c|c|c|c|c|c|c|}
\hline $\begin{array}{l}\text { Isolate } \\
\text { Code }\end{array}$ & 岁 & ச & 트 & $\stackrel{\cup}{0}$ & 郛 & $\sum$ & $\sum_{z}^{u}$ & $\sum$ & 占 & $\stackrel{0}{\underline{1}}$ & అొ & Z & 跣 & $\underset{2}{\longleftarrow}$ & 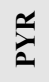 & $\begin{array}{l}\text { Octal } \\
\text { Code }\end{array}$ & Identification \\
\hline P25 & + & - & - & + & + & + & + & + & + & + & + & - & + & + & - & 47766 & S. aureus subsp. aureus \\
\hline ER05 & + & - & + & + & + & + & + & + & + & + & + & - & + & + & - & 57766 & S. aureus subsp. aureus \\
\hline W57 & + & - & + & + & + & + & + & + & + & + & + & - & + & - & - & 57764 & S. aureus subsp. aureus \\
\hline W120 & + & + & + & + & + & + & + & + & + & + & + & - & + & + & - & 77766 & S. aureus subsp. aureus \\
\hline AS103 & + & + & - & + & + & + & + & + & + & + & + & - & + & - & + & 67765 & S. aureus subsp. aureus \\
\hline W15 & + & + & + & + & + & + & + & + & + & + & + & - & + & + & - & 77766 & S. aureus subsp. aureus \\
\hline W11 & - & + & + & + & - & + & + & + & + & + & + & - & + & - & - & 35764 & S. aureus subsp. aureus \\
\hline P94 & + & + & - & + & + & + & + & + & + & + & + & - & + & + & - & 67766 & S. aureus subsp. aureus \\
\hline EY48 & + & + & + & + & + & + & + & + & + & + & + & - & + & + & - & 77766 & S. aureus subsp. aureus \\
\hline AS33 & + & + & + & + & + & + & + & + & + & + & + & + & + & + & - & 77776 & S. aureus subsp. aureus \\
\hline AS67 & + & - & + & + & - & - & - & + & - & - & + & - & - & + & - & 54222 & S. epidermidis \\
\hline EY14 & + & - & - & + & - & - & - & - & + & + & - & - & + & - & + & 44145 & S. epidermidis \\
\hline ER78 & - & - & + & + & - & + & - & + & - & + & + & - & + & - & - & 15264 & S. epidermidis \\
\hline W32 & - & + & - & + & - & - & - & + & - & + & + & - & + & - & - & 24264 & S. epidermidis \\
\hline ER12 & + & - & - & + & - & - & - & + & - & - & + & - & - & + & - & 44222 & S. epidermidis \\
\hline EY17 & - & + & - & + & - & + & + & + & + & + & + & - & + & - & - & 25764 & S. aureus subsp. aureus \\
\hline W37 & - & + & + & + & - & + & + & + & + & + & + & - & + & + & - & 35766 & S. aureus subsp. aureus \\
\hline W72 & + & + & + & + & + & + & + & + & - & + & + & - & + & + & - & 77666 & S. aureus subsp. aureus \\
\hline ER01 & + & + & + & + & + & + & + & + & + & + & + & + & + & + & - & 53565 & S. aureus subsp. aureus \\
\hline ER95 & + & - & - & - & + & + & + & - & - & + & + & - & + & - & - & 43464 & S. aureus subsp. aureus \\
\hline
\end{tabular}

Key: LAT- Latex Agglutination Test, CPG- Colony Pigmentation, NIT- Nitrate, SUC- Sucrose, TRE- Trehalose, MAN- Mannitol, NAG- N-Acetyl Glucosamine, MNS- Mannose, TUR- Turanose, PHO- Alkaline Phosphate, $\beta$ GL- $\beta$ Glucosidase, $\beta$ GN- $\beta$ Glucuronidase, URE- Urease, ARG- Arginine, PYR- Pyrrolidonyl Arylamidase, $\mathrm{W}=$ Wound swab, EY=Eye swab, ER=Ear swab, P=Pus, AS= Aspirate. 


\section{Discussion}

This study evaluated conventional and rapid methods for identification of $S$. aureus from clinical specimens. In our study, Microgen ${ }^{\mathrm{TM}}$ STAPH-ID test, Growth on MSA, DNase agar and catalase test had 100\% sensitivity and specificity respectively. Haemolysis on Blood agar had $77.53 \%$ sensitivity and $64.29 \%$ specificity while Gram staining reported $69.00 \%$ sensitivity and $44.64 \%$ specificity. Coagulase test was $100 \%$ sensitive and $94.92 \%$ specific. Microgen ${ }^{\text {TM }}$ STAPH-ID test also identified three coagulase negative $S$. aureus (CoNS) isolates that had been missed by coagulase test, although they were identified by other tests. Altered colonial morphology and negative reaction could have been due to subsequent sub-culturing before testing. This observation suggests that Microgen TM STAPH-ID test may be more specific than coagulase test for identifying $S$. aureus. Hence, to boost the specificity of coagulase test, sequel tests should be conducted on fresh isolates. Furthermore, it is conceivable that misleading results may occur if single tests such as haemolysis on Blood agar are used, particularly if there is a mixed growth, hence, the use of MSA and DNase agar are advised. Procop et al (13) suggested that routine culture with standard agar plates continue to be used to identify cultures that contain mixtures of bacteria. Microgen ${ }^{\mathrm{TM}}$ STAPH-ID test, Growth on MSA, DNase agar and catalase test were the most reliable tests with $100 \%$ sensitivity and specificity. This is in line with Najjuka et al (12) who reported that growth on MSA was the best at identifying $S$. aureus (though with lower sensitivity and specificity of $94 \%$ and $79 \%$ respectively) and recommended simultaneous use of all the three tests (beginning with growth on MSA, DNase and then Tube coagulase) for identification of $S$. aureus. Microgen $^{\text {TM }}$ STAPH-ID test was reliable in this study, but also relatively expensive; hence, its extensive use for routine identification of $S$. aureus in a developing country like Nigeria is a great challenge.

\section{Conclusion}

This study reveals that routine conventional tests are reliable for identification of $S$. aureus in resource limited areas. There is however, no single test that can guarantee reliable results, hence the need for sequel testing using mannitol salt agar, DNase agar, then coagulase test.

\section{References}

[1] F. Menichetti, "Current and Emerging Serious GramPositive Infections", Clinical Microbiology and Infections, vol. 11(3), pp. 22-28, 2005, doi:10.1111/j.14690691.2005.01138.x. PMID 15811021.
[2] D. T Durack, A. S Lukes, D. K Bright, S. Duke, New criteria for diagnosis of infective endocarditis: utilization of specific echocardiographic findings, Am J Med, vol.96(3), pp. 200209, 1994.

[3] F. Martineau, F. J. Picard, P. H. Roy, M. Ouellette, M. G. Bergeron, Species-specific and ubiquitous-DNA-based assays for rapid identification of Staphylococcus aureus, J Clin Microbiol, vol. 36(3), pp. 618-623, 1998.

[4] C. S. S Bello, A. Qahtani, Pitfalls in the routine diagnosis of Staphylococcus aureus, Afr J Biotech, vol. 4(1), pp. 83-86, 2006.

[5] J.N.M. Mugalu, S. Kiguli, D. H Kaddu-Mulindwa, Aetiology, risk factors and immediate outcome of bacteriologically confirmed neonatal septicaemia in Mulago hospital, Uganda, Afr Health Sc, vol. 6(2), pp. 120-126, 2006.

[6] W. E. Kloos, T. L. Bannerman, Staphylococcus and Micrococcus, In: Manual of Clinical Microbiology, 6th edn,. Edited by P. R. Murr, pp. 282-298, 1995.

[7] G. H. Chapman, The significance of sodium chloride in studies of staphylococci, J Bacteriol, vol. 50, pp. 201-203, 1945.

[8] J. P. Duguid, Staphylococcus: cluster-forming Gram-positive cocci. In: Mackie and McCartney Practical Medical Microbiology, 13th edn, Edited by J. G. Colle, J. P. Duguid, A. G. Fraser \& B. P. Marmion. New York: Churchill Livingstone, pp.303-316, 1989.

[9] P. Jayaratne, C. Rutherford, Detection of methicillinresistant Staphylococcus aureus (MRSA) from growth on mannitol salt oxacillin agar using PCR for nosocomial surveillance, Diagn Microbiol Infect Dis, vol. 35, pp. 13-18, 1999.

[10] A. Simor, J. Goodfellow, L. Loiue, M. Loiue, Evaluation of a new medium, oxacillin resistance screening agar base, for the detection of methicillin-resistant Staphylococcus aureus from clinical specimens. J Clin Microbiol, vol. 33, pp. 3422, 2001.

[11] P. M. Zadik, S. Davies, S. Whittaker, C. Mason, Evaluation of a new selective medium for methicillin-resistant Staphylococcus aureus, J Med Microbiol, vol. 50, pp. 476479, 2001.

[12] F. C. Najjuka, D. P. Kateete, C. N Kimani, F. A. Katabazi, A. Okeng, M. S Okee, A. Nanteza, M. L Joloba, Identification of Staphylococcus aureus: DNase and Mannitol salt agar improve the efficiency of the tube coagulase test, Annals of Clinical Microbiology and Antimicrobials, vol. 9, pp. 23, 2010, doi:10.1186/1476-0711-9-23

[13] G. W. Procop, N. K. Shrestha, M. J. Tuohy, G. S. Hall, C. M Isada, Rapid Identification of Staphylococcus aureus and the mecA Gene from BacT/ALERT Blood Culture Bottles by Using the LightCycler System, J Clin Microbiol, vol. 40(7), pp. 2659-2661, 2002, doi: 10.1128/JCM.40.7.26592661.2002 PMCID: PMC120611 